\title{
The Polycystic Ovary Syndrome and the Metabolic Syndrome: A Possible Chronobiotic-Cytoprotective Adjuvant Therapy
}

\author{
Eduardo Spinedi $\mathbb{D}^{1}$ and Daniel P. Cardinali ${ }^{2}$ \\ ${ }^{1}$ Centre for Experimental and Applied Endocrinology (CENEXA, UNLP-CONICET-FCM), CEAS-CICPBA, \\ La Plata Medical School, La Plata, Argentina \\ ${ }^{2}$ BIOMED-UCA-CONICET and Department of Teaching and Research, Faculty of Medical Sciences, \\ Pontificia Universidad Católica Argentina, Buenos Aires, Argentina \\ Correspondence should be addressed to Eduardo Spinedi; spinedi@cenexa.org
}

Received 18 April 2018; Accepted 28 June 2018; Published 25 July 2018

Academic Editor: Maria C. Meriggiola

Copyright ( 2018 Eduardo Spinedi and Daniel P. Cardinali. This is an open access article distributed under the Creative Commons Attribution License, which permits unrestricted use, distribution, and reproduction in any medium, provided the original work is properly cited.

\begin{abstract}
Polycystic ovary syndrome is a highly frequent reproductive-endocrine disorder affecting up to $8-10 \%$ of women worldwide at reproductive age. Although its etiology is not fully understood, evidence suggests that insulin resistance, with or without compensatory hyperinsulinemia, and hyperandrogenism are very common features of the polycystic ovary syndrome phenotype. Dysfunctional white adipose tissue has been identified as a major contributing factor for insulin resistance in polycystic ovary syndrome. Environmental (e.g., chronodisruption) and genetic/epigenetic factors may also play relevant roles in syndrome development. Overweight and/or obesity are very common in women with polycystic ovary syndrome, thus suggesting that some polycystic ovary syndrome and metabolic syndrome female phenotypes share common characteristics. Sleep disturbances have been reported to double in women with PCOS and obstructive sleep apnea is a common feature in polycystic ovary syndrome patients. Maturation of the luteinizing hormone-releasing hormone secretion pattern in girls in puberty is closely related to changes in the sleep-wake cycle and could have relevance in the pathogenesis of polycystic ovary syndrome. This review article focuses on two main issues in the polycystic ovary syndrome-metabolic syndrome phenotype development: (a) the impact of androgen excess on white adipose tissue function and (b) the possible efficacy of adjuvant melatonin therapy to improve the chronobiologic profile in polycystic ovary syndrome-metabolic syndrome individuals. Genetic variants in melatonin receptor have been linked to increased risk of developing polycystic ovary syndrome, to impairments in insulin secretion, and to increased fasting glucose levels. Melatonin therapy may protect against several metabolic syndrome comorbidities in polycystic ovary syndrome and could be applied from the initial phases of patients' treatment.
\end{abstract}

\section{Introduction}

Polycystic ovary syndrome (PCOS) is a highly frequent reproductive-endocrine disorder detected in up to $8-10 \%$ of women at reproductive age worldwide. Due to its heterogeneous nature, different criteria have been established in the course of several years in order to lead to a more precise PCOS diagnosis [1-5]. Presently, accepted criteria indicate that PCOS patients are characterized by oligomenorrhea or amenorrhea, hyperandrogenism, and/or hyperandrogenemia, with enlarged ovary volume full of cystic ultrasound images [1].
Although the etiology of PCOS is not fully understood, evidence suggests that insulin resistance (IR), with or without compensatory hyperinsulinemia, contributes to inhibit liver sex hormone-binding globulin (SHBG) production and to stimulate ovarian/adrenal androgen secretion. Dysfunctional white adipose tissue (WAT) has been identified as a major contributing factor for IR in PCOS. Environmental and genetic/epigenetic factors may also play relevant roles in PCOS development $[6,7]$.

The dysmetabolic aspect of PCOS has recently gained much attention. Several features of metabolic disturbances, particularly IR and hyperinsulinemia, have been observed 
in most women with PCOS [8]. Therefore, overweight and/or obesity (specifically, enlarged visceral fat) are very common in women with PCOS, thus indicating that some PCOS and metabolic syndrome (MS) female phenotypes share common characteristics [9].

MS is a cluster of endocrine-metabolic dysfunctions including IR, overweight/central obesity, dyslipidemia, hypertension, and high risk of cardiovascular disease [10]. In this regard, studies confirm that MS is more common among PCOS patients due to the higher prevalence of IR and hyperadiposity (visceral) in these women [11, 12]. Moreover, PCOS patients have an elevated risk of MS [12], indicating that WAT dysfunction is a highly prevalent and common feature in the PCOS-MS phenotype $[4,13]$.

Although not yet deeply investigated, sleep disturbances have been reported to double in women with PCOS. Indeed, PCOS patients displayed increased difficulty in falling to sleep and staying asleep [14], with excessive daytime somnolence being higher in sleep-disordered than in not sleepdisordered PCOS women [15]. In this regard, obstructive sleep apnea is a common feature in adult [16] and adolescent [17] PCOS patients suffering multiple comorbidities, such as IR and hyperadiposity. Significant chronodisruption occurs in patients suffering from obstructive sleep apnea as evidenced by a disrupted melatonin circadian rhythm [18]. A clear relationship between sleep disturbances and reproductive dysfunction has also been noticed in non-PCOS women: those sleeping less than $6 \mathrm{~h}$ daily have shorter or longer menstrual cycles [19].

Experiments using neonatally androgenized female rats (the Barraclough PCOS model) indicated an altered pattern of hypothalamic luteinizing hormone-releasing hormone (LHRH) and pituitary LH/FSH contents directly related to changes in the pulsatile pattern of LH/FSH release [20]. In this regard, maturation of the LHRH secretion pattern in girls across puberty has been reported to be closely related to changes in the sleep-wake cycle and is suggested to be relevant in the pathogenesis of PCOS [21].

Overall metabolic-reproductive-cardiovascular risk associated with PCOS urges for a more broadly specific therapeutic approach in medical care of PCOS patients. This short review article focuses on two main issues in PCOS-MS phenotype development: (a) the impact of androgen excess on WAT function and (b) a possible adjuvant melatonin therapy to improve the chronobiologic profile in PCOS individuals.

\section{Cooperative Effect of Androgens on White Adipose Tissue Dysfunction}

Adipose tissue mass and distribution pattern display clear gender/sex dimorphism [22, 23]. Whereas men have greater predisposition to accumulate visceral adipose tissue (VAT) (known as android distribution) [23], women accumulate WAT in the gluteofemoral position (known as gynoid distribution) [22]. VAT mass expansion is associated with a higher risk of type 2 diabetes mellitus and cardiovascular disease [24]. The relationship between blood androgen levels and WAT function in women seems to be more complex. It is accepted that androgen excess is associated with central obesity, although some studies deny this assumption. PCOS phenotypes often have hyperandrogenemia associated with IR and accumulate WAT mass [25, 26]. Testosterone is able to directly induce IR in adipocytes [27], in part by decreasing cellular glucose uptake [28].

Studies performed on the preadipocyte cell line 3T3-L1 and on multipotent cell lines (C3H10T1/2) indicate that testosterone inhibits cell proliferation and differentiation to mature adipocytes [29-31]. A similar inhibitory testosterone effect is found in human adipocyte precursor cells (APCs) from distinct WAT pads. It is possible that the antiadipogenic action of androgens is due to the inhibiting activity of PPAR $-\gamma 2$ and C/EBP- $\alpha$ functions; interestingly, induction of APCs to differentiate results in increased AR expression [32]. Moreover, it is widely accepted that inhibition of the adipogenic process results in hypertrophic WAT mass expansion due to enlargement of the local adipocyte size [33]. Thus, in spite of its inhibitory effect on adipogenesis, it seems feasible that testosterone could induce WAT (VAT) pad mass expansion by increasing the cell size (hypertrophic pad mass expansion). Most studies of androgen effects on the adipogenic process have focused on the terminal phase of the process, then it remains to be determined whether testosterone acts on other stages of adipogenesis (e.g., by influencing APC number and competency).

It must be taken into account that hypertrophy expansion of WAT mass, as it occurs in an androgen excess endogenous milieu, is due to the large size of local adipocytes that release large amounts of proinflammatory adipocytokines [such as leptina (LEP), resistin, tumor necrosis factor alpha (TNF $\alpha)$, plasminogen activator inhibitor type-1 (PAI-1), interleukin-1 (IL-1), and IL-6, among others]. These substances are highly capable of diminishing tissue insulin sensitivity and promoting oxidative stress (OS). Moreover, hypertrophic WAT cells did also release few amounts of adiponectin (ADIPOQ), an insulin-sensitizing adipokine, thus worsening local (white adipocyte) and peripheral tissue insulin sensitivity.

Interestingly, studies in the rodent neonatal androgenization model of PCOS clearly showed that early transient testosterone excess in female rats resulted in an adult phenotype characterized by several endocrine-metabolic dysfunctions [34]. Indeed, these rats showed altered WAT (parametrial tissue-pad) functionality, such as enlarged pad mass, replete of large (insulin-resistant) adipocytes and containing very low ADIPOQ protein [34]. Moreover, their isolated WAT adipocytes did release excess of LEP and resulted to be highly resistant to insulin-stimulated LEP secretion [34]. These dysfunctional PCOS rats were peripherally characterized by increased levels of LEP, PAI-1, and nonesterified fatty acids and decreased ADIPOQ concentration [34]. Their responses to an ip glucose tolerance test indicated (by analyzing the area under the curve values) that although they were still fully able to manage glycemias (normal glucose tolerance), this occurred at the expenses of compensatory hyperinsulinemias, whereas adiponectinemias remained very low [34]. Some of these altered functions are shown in Figure 1. The alterations indicate that a phenotype shifted towards an overall state of IR and inflammation. Relevantly, 
Parametrial adipose tissue characteristics

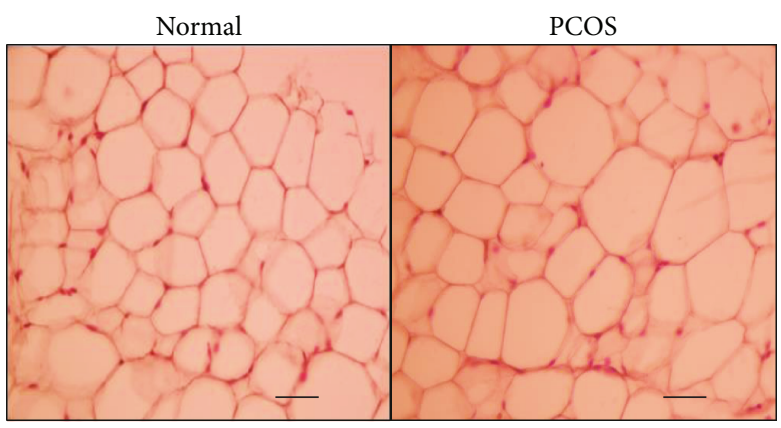

(a)
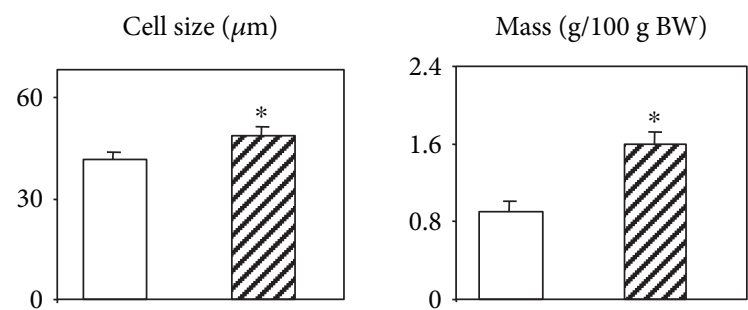

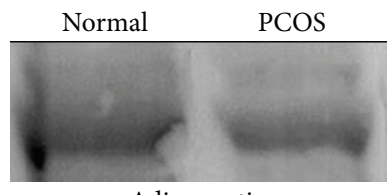

Adiponectin

(b)

Cell leptin

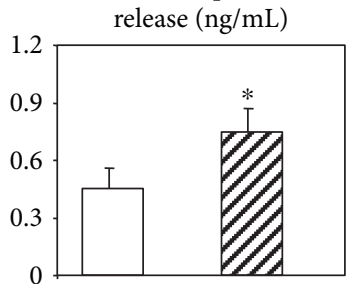

FIGURE 1: White adiposity characteristics in normal and PCOS adult rats. Representative images of parametrial adipose tissue pads stained with hematoxylin and eosin (a). Lower 3-diagram panel showing data from parametrial pad adipocyte (in left-right order) size, mass, and in vitro leptin secretion. Finally, parametrial pad adiponectin protein content (Western blot) (b). Magnification $\times 400$; scale bars: $50 \mu \mathrm{m}$. ${ }^{*} P<0.05$ versus respective normal-group values (adapted from Alzamendi et al. [34]).

neonatal treatment with flutamide, a nonsteroidal antagonist of the androgen receptor, induced a decrease in the peripheral levels of leptin [35]. Collectively, these data indicate that early treatment with testosterone increases susceptibility to MS [36] development and, conversely, that flutamide treatment improves this condition. These observations strongly support that testosterone exerts specific receptor-mediated effects [37], the androgen receptor being expressed in both white adipocytes and APCs [38]. However, the level of androgen receptor expression differs among WAT depots. VAT has higher receptor expression levels than subcutaneous fat deposits $[32,38,39]$, which could explain in part the differential actions of testosterone on different adipose tissue depots.

Altered lipolysis/lipogenesis balance contributes to the increase in lipid storage in adipose cells and therefore to the development of unhealthy (hypertrophic) VAT mass expansion. As mentioned above, androgen excess is per se a clear inducing factor of white adipocyte hypertrophy, and these cells overproduce (enhanced synthesis and secretion) proinflammatory adipokines (including angiotensinogen and free fatty acids), resulting in a local inflammatory state. Large adipocytes are indeed insulin-resistant (IR) and characterized by high cell reticulum endoplasmic oxidative stress (REOS) content, enhanced lipolytic activity, cell hypoxia, and apoptosis. Moreover, large IR adipocytes are not well recognized by the immune system, and, as a consequence, macrophages react against to and infiltrate dysfunctional WAT, thus worsening the inflammatory state. These alterations in PCOS women, in turn, affect multiple organ functions, namely, at liver [40], muscle [41], endocrine pancreas [42], and endothelium [43] levels, thus compromising cardiovascular function (hypertension and atherogenesis) [44] (Figure 2).

Regarding ovarian function, recent studies from one of our laboratories using the PCOS rat phenotype indicated a clear ovary dysfunctional folliculogenesis [45] as indicated by the number of secondary and atretic stage follicles; indeed, a 3-fold lower number and a 5-fold higher number, respectively, were noticed in the PCOS rat phenotype than in the normal rat (Figure 3(a)). Moreover, ovary images in normal animals fully correspond with expected characteristics (e.g., antral cavity, corpus luteum, oocyte, granulosa cells, and internal and external theca cells) (Figure 3(b)), whereas those from PCOS rats displayed dysfunctional characteristics compatible with a large antral cavity in a cystic follicle and showed decreased granulosa and theca (internal and external) cell layers (Figure 3(b)). This misprogramming in carbohydrate metabolism and dyslipidemia (e.g., a prediabetic state) as well as the inflammatory state indicate that the PCOS rat phenotype is highly prone to developing cardiovascular disease and reproductive dysfunction (abnormal folliculogenesis). Therefore, subfertility/infertility and/or poor pregnancy outcome (early abortion and/or preeclampsia) could ensue.

\section{Chronobiology in PCOS}

As mentioned, some PCOS phenotypes carry a significant risk for metabolic disturbances including MS, prediabetes, 


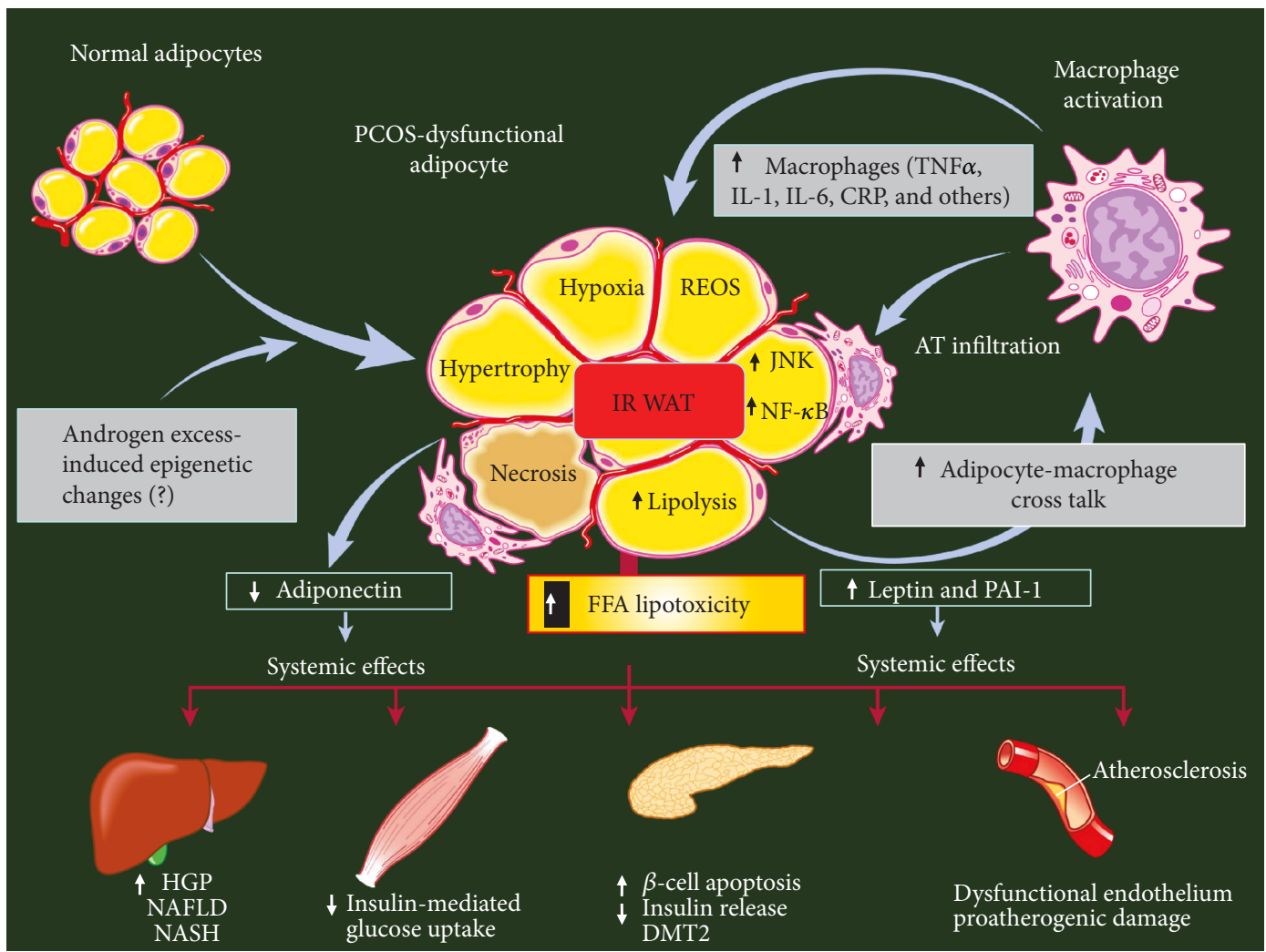

FIGURE 2: White adipose tissue (WAT) and inflammation: endocrine-metabolic consequences. A combination of genetic background and endogenous androgen excess could induce WAT mass hypertrophic expansion associated with macrophage infiltration, leading to an abnormal pattern of adipokine secretion. Enhanced WAT-derived leptin release, in turn, impairs tissue sensitivity to insulin (insulin resistance (IR)). Prolonged hyperleptinemia could induce long-form leptin receptor (ObRb) downregulation, namely, at the pancreatic ( $\beta$ - and $\alpha$-cell) level, thus impairing its negative feedback mechanism on insulin (and glucagon) secretion; moreover, increased release of proinflammatory signals (TNF, IL-1, IL-6, and C-reactive protein (CRP), among others) worsens several functions. In fact, overall WAT dysfunction promotes multiple endocrine-metabolic dysfunctions, such as generalized IR, enhanced reticulum endoplasmic oxidative stress (REOS), enhanced lipolytic activity, cell hypoxia, and apoptosis. These alterations, in turn, affect multiple peripheral organs, namely, liver, muscle, endocrine pancreas, and endothelium functions. FFA: free fatty acid; JUNK: Janus kinase; NF- $k \mathrm{~B}$ : nuclear factor- $\kappa \mathrm{B}$; HGP: hepatic glucose production; NAFLD: nonalcoholic fatty liver disease; NASH: nonalcoholic steatohepatitis; DMT2: diabetes mellitus type 2 (adapted from Pagano et al. [108]).

and type 2 diabetes and an intrinsic prooxidant state resulting from imbalance between excessive oxidant production in the presence of limited antioxidant defence. MS comprises many risk factors for cardiovascular disease including hyperinsulinemia, glucose intolerance, dyslipidemia, hyperadiposity/obesity, and elevated blood pressure. MS prevalence ranges from 15 to $30 \%$ depending on the world region considered $[46,47]$. A 1.5 - to 2.5 -fold increase in cardiovascular mortality occurs when MS is present, representing one of the major public health problems at this time.

In the last decade, the understanding of the cellular and molecular events that contribute to MS development has increased considerably. One basic function apparently heavily influenced by obesity and metabolic disease is the internal timing system [48-50]. The correlation between increased occurrence of obesity and the ubiquity of modern social habits, such as light at night, unusual meal times, and irregular sleep/wake schedules, all encompassed by a " $24 / 7$ " lifestyle, strongly suggests that impairment of sleep and the circadian system is involved in the etiology of MS.
Several clinical surveys have shown increased prevalence of MS in night-shift workers, indicating that artificial lighting may contribute to increased prevalence of metabolic disorders [51-55].

Because melatonin, as a chronobiotic/cytoprotective agent, has a special place in prevention and treatment of MS [52-54], its possible therapeutic utility in PCOS has been considered. Low levels of melatonin at night have been linked to metabolic abnormalities such as insulin resistance and type 2 diabetes mellitus. Moreover, the suppression of nocturnal melatonin by light exposure at night has been associated with several pathologies comprising MS [56-58].

Melatonin is measurable in human preovulatory follicular fluid and may play a role in regulating ovarian steroidogenesis, folliculogenesis, and oocyte maturation [59]. Melatonin can protect follicles against oxidative stress and may rescue follicles from atresia, thereby promoting correct follicular maturation and, ultimately, ovulation [60].

Table 1 summarizes results supporting a therapeutic role of melatonin in PCOS. In rats, the reduction of circulating 


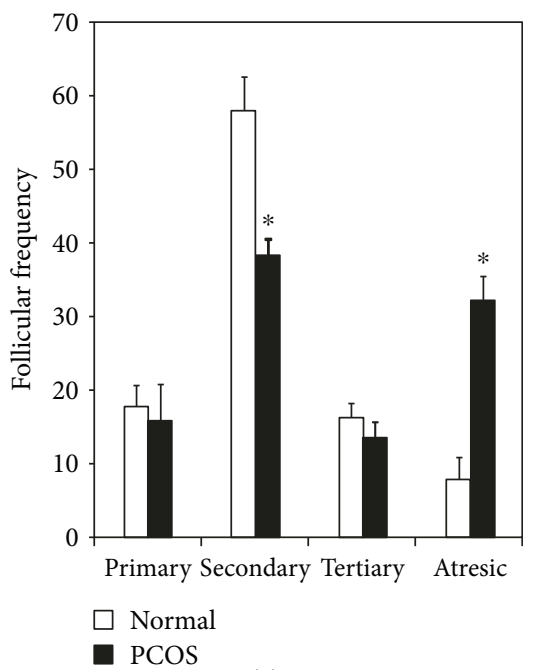

(a)

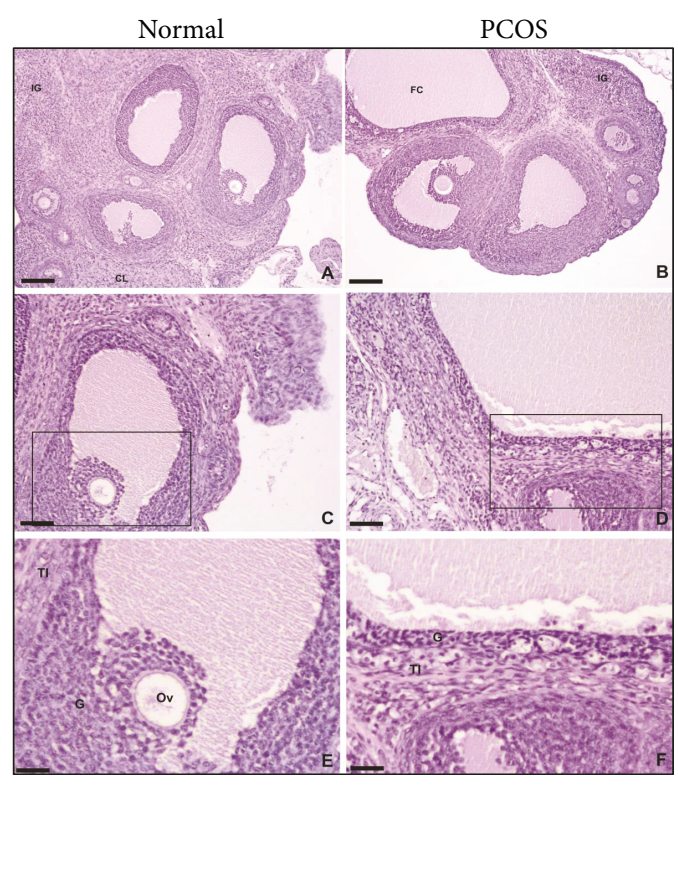

(b)

FIGURE 3: Follicular developmental stage of the ovaries obtained from normal and PCOS adult rats (a). Values are expressed in percentages. ${ }^{*} P<0.05$ versus normal-group values. Representative images (b) of the ovaries from normal (A, C, and E) and PCOS (B, D, and F) adult rats showing ovarian structures at different magnifications: (A) and (B): 10x (bars: $100 \mu \mathrm{m}$ ); (C) and (D): 20x (scale bars: $50 \mu \mathrm{m}$ ); (E) and (F): 40x (scale bars: $25 \mu \mathrm{m}$ ). CL: corpus luteum; IG: interstitial glands; FC: follicular cyst; G: granulosa; TI: theca interna; Ov: ovocyte (adapted from Ongaro et al. [45]).

TABle 1: Relevance of melatonin in PCOS.

\begin{tabular}{|c|c|}
\hline Observation & Reference/s \\
\hline Significantly higher secretion of melatonin in PCOS women & [64-68] \\
\hline Family association study between melatonin receptor gene polymorphisms and PCOS & [77-79] \\
\hline Supplementation of in vitro culture medium with melatonin improved IVF outcome in PCOS & {$[80]$} \\
\hline $\begin{array}{l}\text { Melatonin and myo-inositol enhanced, synergistically, oocyte, and embryo quality and } \\
\text { improved in vitro fertilization of patients with PCOS }\end{array}$ & [81] \\
\hline Melatonin treatment restores menstrual cyclicity in women with PCOS & {$[82]$} \\
\hline $\begin{array}{l}\text { Treatment of preinvasive endometrial cancer in women with PCOS using female sexual hormones in } \\
\text { combination with melatonin, antidiabetic, antidopaminergic, and antiserotonin therapy favorably influenced } \\
\text { female sexual hormone profile and lipid metabolism and caused the restoration of normal endometrium }\end{array}$ & [83] \\
\hline Melatonin treatment ameliorated oxidative stress and inflammatory parameters of obese women & {$[84]$} \\
\hline Reduced fat mass and increased lean mass in response to 1 year of melatonin treatment in postmenopausal women & {$[85]$} \\
\hline Protective effects of melatonin against metabolic and reproductive disturbances in rodent models of PCOS & [104-107] \\
\hline
\end{tabular}

melatonin levels after pinealectomy induces the development of some characteristics of PCOS. Published data indicate that the direct effect of melatonin on follicular steroid production is complex and may depend on the cell type (theca cell or granulose cell), duration of treatment (acute or long-term response), experimental model (cell culture or follicle culture), species, and dose [61]. Melatonin may directly suppress follicular (thecal) steroidogenesis at an early stage in the steroid synthesis pathway by blocking the expression of steroidogenic acute regulatory protein, which facilitates translocation of cholesterol across the intermembrane space into the inner membrane to be cleaved into pregnenolone. Treatment of rats with melatonin can reduce obesity, type 2 diabetes, and hepatic steatosis $[62,63]$, and in several animal models of hyperadiposity melatonin injection normalized most observed alterations and corrected the altered biochemical proinflammatory profile.

A significantly higher excretion of 6-sulfatoxymelatonin, the major excretory metabolite of melatonin, was reported in patients with PCOS [64-66]. Other studies later described higher serum melatonin concentrations associated with ovarian intrafollicular deficiency of melatonin in patients 
with PCOS $[67,68]$. Regardless of that, the intrafollicular melatonin concentrations are lower in PCOS than in controls [61]. The high circulating levels of melatonin in PCOS may be a feedback response to the deficient levels of melatonin in the ovary [61]. High levels of melatonin in the follicular fluid is essential for follicle growth, ovulation, and oocyte quality, whereas reduced follicular melatonin concentrations may be responsible for anovulation and poor oocyte quality in PCOS.

Melatonin communicates nightly timing cues through activation of two $G$ protein-coupled receptors, that is, melatonin receptor 1 (MT1) and melatonin receptor 2 (MT2) [68]. Both MT1 and MT2 have been shown to activate several signalling pathways, most notably the Gi/cAMP and $\mathrm{Gq} /$ phospholipase $\mathrm{C} / \mathrm{Ca}^{2+}$ pathways. These receptors are expressed in many different peripheral tissues such as the ovary and modulate multiple aspects of human physiology [69].

Genome-wide association studies have shown that polymorphisms in the genes encoding human melatonin receptors (MTNR1A and MTNR1B) are involved in the pathogenesis of type 2 diabetes mellitus [70-76]. By resequencing the coding region of the MTNR1B gene coding for the MT2 receptor, variants have been identified and functionally characterized. Corresponding mutants with impaired receptor signalling are strongly associated with diabetic risk, indicating that loss of melatonin receptor function is positively associated with disease risk.

Variants in MTNR1B (MT2) have been linked to impairments in both insulin secretion and increased fasting glucose levels, and variants in MTNR1A (MT1) have been shown to be associated with increased risk of developing PCOS [77-80]. Polymorphisms rs2119882 in the MTNR1A gene and rs10830963 in the MTNR1B gene were proposed to have a common causative role in the pathogenesis of PCOS. However, in a recent study to investigate whether an association exists between these two single-nucleotide polymorphism variants and PCOS, an association was detected only between rs2119882 in the MTNR1A gene and PCOS [77]. Collectively, genetic data provide a basis for further studies of the MTNR gene in the etiology of PCOS.

Melatonin may directly affect ovarian function: it is concentrated in human ovarian follicles relative to the level in plasma, and it alters granulosa cell steroidogenesis and follicular function in humans [60]. However, only a few studies have been published on melatonin potential as a therapeutic agent in humans in the PCOS.

Two of them relate to improvement of in vitro fertilization of patients with PCOS. The supplementation of in vitro culture medium with melatonin improved in vitro fertilization outcome in PCOS [81] while melatonin and myo-inositol enhanced, synergistically, oocyte and embryo quality and improved in vitro fertilization of patients with PCOS [82]. These findings suggest that the addition of melatonin to in vitro fertilization media may improve the cytoplasmic maturation of immature oocytes.

In an open-label study including 40 normal-weight women with PCOS, ultrasound pelvic examinations, hirsutism score evaluation, hormone profile assays, oral glucose tolerance test, and lipid profile at baseline and after a 6-month administration of $2 \mathrm{mg}$ fast release melatonin po daily at bedtime were recorded [83]. Melatonin treatment significantly decreased serum androgen and $17 \alpha$-hydroxyprogesterone levels and augmented serum FSH and antiMullerian hormone serum levels. Almost 95\% of participants experienced an amelioration of menstrual cycle disruption. No significant changes occurred in glucoinsulinemic and lipid parameters after treatment except a significant decrease in low-density lipoprotein cholesterol.

Treatment of preinvasive endometrial cancer in women with PCOS using melatonin in combination with female sexual hormones and antidiabetic, antidopaminergic, and antiserotoninergic therapy favorably influenced female sexual hormone profile and lipid metabolism and caused restoration of normal endometrium [84]. Indeed, melatonin treatment ameliorated oxidative stress and inflammatory parameters of obese women [85] and reduced fat mass and increased lean mass in postmenopausal women [86].

Therefore, the data in Table 1 agree with many studies now supporting the beneficial role of melatonin in patients with MS. Melatonin treatment ameliorates MS in obese patients $[87,88]$ as well as in bipolar and schizophrenic patients after treatment with second-generation antipsychotics [89-91]. Melatonin administration normalizes MS in elderly hypertensive patients [92] and improves enzyme profile in patients with alcoholic liver steatosis [88, 89]. Using melatonin and zinc acetate, when employed alone or in combination with metformin, improved glycemic control in type 2 diabetic patients [93], and an inverse relationship between urinary 6-sulfatoxymelatonin excretion and insulin levels versus insulin resistance was reported in healthy women in the Nurses' Health Study cohort [94]. It must be noted however that there are results that deny the capacity of melatonin to improve glucose tolerance and to reduce insulin resistance in humans. Melatonin administration decreased glucose tolerance, already in nondiabetic young individuals [95-97]. Although the results summarized in Table 1 suggest that melatonin therapy may be beneficial for patients with PCOS, more studies are obviously needed to evaluate an appropriate time/duration of treatment/dose relationship for administration of melatonin.

\section{Concluding Remarks}

Many metabolic-reproductive alterations associated with PCOS are closely dependent on WAT dysfunction, particularly at the VAT pad level. However, the increase in VAT pad mass per se is not an unequivocal indication of VAT dysfunction, whereas development of enlarged local adipocytes is indeed a key factor. Androgen excess is able to induce an imbalance between white adipocyte hypertrophy and hyperplasia, towards enlarged (IR) adipocytes and consequent VAT dysfunction and inflammation. Many factors regulate normal VAT mass expansion. In this review, we ponder the influence of testosterone, a sex steroid hormone in excess (hyperandrogenemia and/or hyperandrogenism) in PCOS phenotypes. Factors modulating adipogenesis/ inflammation could become new therapeutic targets to 


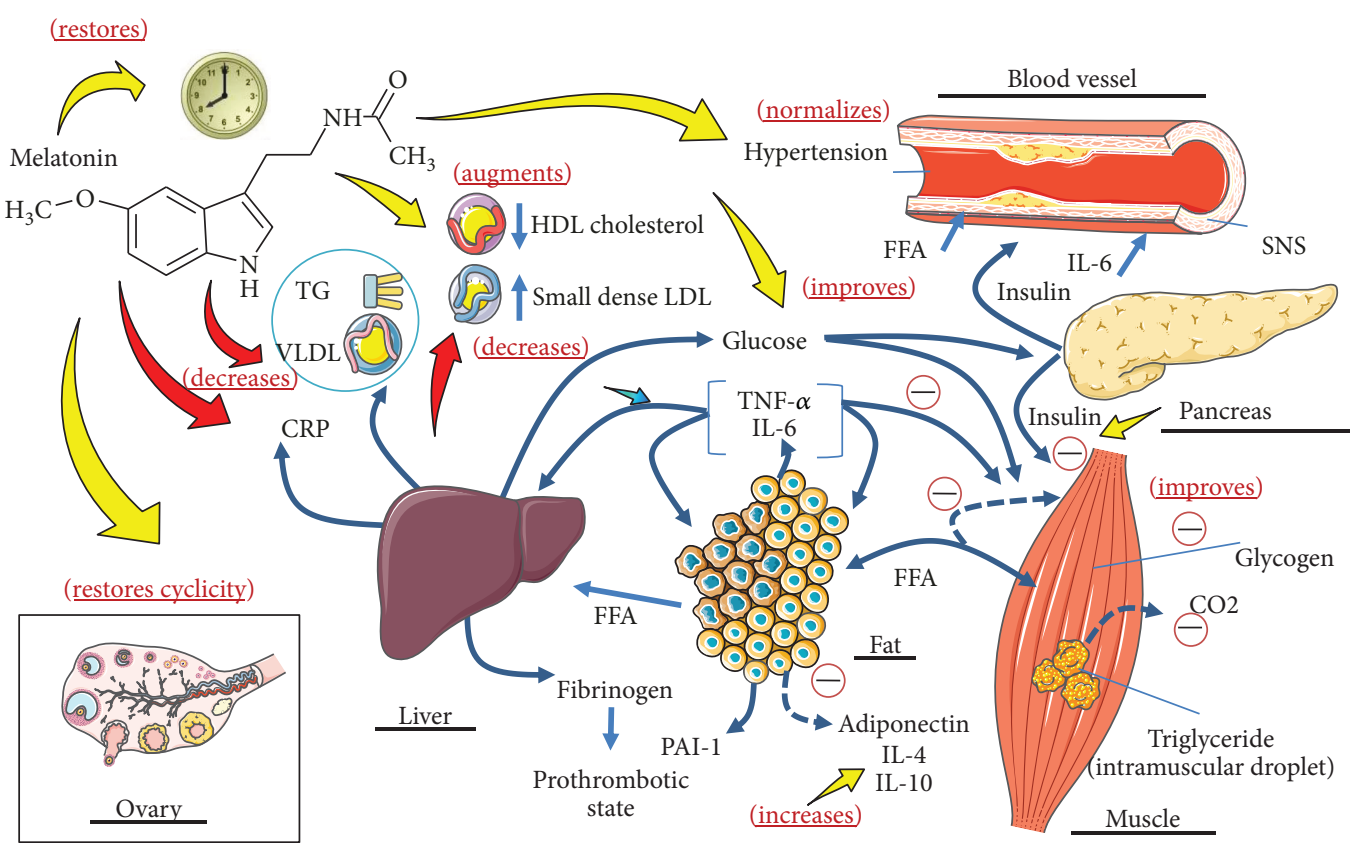

FIGURE 4: Effect of melatonin in PCOS associated with metabolic syndrome. Melatonin normalizes high blood pressure (BP) and circulating indexes of inflammation. It also improves insulin sensitivity and restores disrupted circadian rhythms. Melatonin directly affects ovarian function: it is concentrated in human ovarian follicles in relation to the level in plasma, and it improves granulosa cell steroidogenesis and follicular function in humans (adapted from Reiter et al. [61]).

counteract endocrine-metabolic-reproductive dysfunction, favoring healthy WAT mass expansion and counteracting hypertrophic adiposity-associated dysfunctions.

According to the Rotterdam ESHRE/ASRM-Sponsored PCOS Consensus Workshop Group, it was concluded that PCOS is a syndrome of ovarian dysfunction along with the cardinal feature hyperandrogenism and polycystic ovary (PCO) morphology [1]. However, because PCOS remains a syndrome, not a single diagnostic criterion is sufficient for clinical diagnosis. There are many other clinical manifestations that may be included, such as menstrual irregularities, signs of androgen excess, obesity, insulin resistance, elevated serum levels of LH, increased risk of type 2 diabetes [98], and cardiovascular events [99]. Clinical management of PCOS should include rigorous lifestyle modifications, insulin therapy, and drug treatments that promote insulin sensitization (such as metformin) and insulin secretion (such as glibenclamide), dipeptidyl peptidase-4 inhibitors, sodium glucose cotransporter 2 inhibitors [100], and antihyperlipidemic therapy [101]. In general, these approaches are designed to manage symptoms of insulin resistance/ $\beta$-cell dysfunction and dyslipidemia and are used either alone or in combination. Drug therapies are expensive worldwide and in some cases have been associated with adverse secondary events including pancreatitis, hypoglycemia, and osteoporosis $[102,103]$. Therefore, a need remains for new and costeffective pharmacotherapies for diabetes presenting limited additional health risks.

Although overweight/obesity is preventable, its prevalence is continuously increasing worldwide, and because it is frequently associated with other cardiovascular risk factors and high mortality, obesity has become an important public health problem and a heavy socioeconomic burden for society as a whole. Environmental factors, hormone excess (e.g., androgen), or stressors of so-called contemporary "24/7" societies have pronounced effects on metabolism producing circadian clock disruption. Further, people whose work involves irregular time schedules and forced exposure to bright light at night (night/shift workers) show significant disruptions in sleep architecture and increased prevalence of MS. These lines of evidence indicate that the body's system fails to adjust properly to environmental and/or stressor changes disrupting overall metabolic homeostasis.

Melatonin may provide an innovative strategy in PCOS by combining its chronobiotic effect on circadian rhythm with cytoprotective properties. Indeed, melatonin protects against several MS comorbidities in PCOS, such as diabetes and concomitant oxy-radical mediated damage, inflammation, microvascular disease, atherothrombotic risk, and ovary dysfunction. Melatonin may therefore have a place from the initial phases of PCOS treatment. Its high safety profile and reduced toxicity distinguishes it from many pharmaceutical agents used in PCOS patients (Figure 4). In conclusion, an appropriately classical pharmacological treatment combined with melatonin should be considered in PCOS individuals to restore endocrine-metabolic and reproductive functions.

\section{Conflicts of Interest}

The authors declare that there is no conflict of interest. 


\section{Authors' Contributions}

Eduardo Spinedi and Daniel P. Cardinali contributed equally to this work.

\section{Acknowledgments}

The authors wish to thank Susan H. Rogers for editing and correcting this manuscript. The authors are Research Career Awardees from the National Research Council of Argentina (CONICET). Studies carried out by authors were supported by grant PICT (2007-01045 and 2012-0984) from the ANPCyT, Argentina and, the Swiss Foundation for Research on Endocrinology, Diabetes and Metabolism (FPREDM052015).

\section{References}

[1] Rotterdam ESHRE/ASRM-Sponsored PCOS Consensus Workshop Group, "Revised 2003 consensus on diagnostic criteria and long-term health risks related to polycystic ovary syndrome," Fertility and Sterility, vol. 81, no. 1, pp. 19-25, 2004.

[2] R. Azziz, E. Carmina, D. Dewailly et al., "The Androgen Excess and PCOS Society criteria for the polycystic ovary syndrome: the complete task force report," Fertility and Sterility, vol. 91, no. 2, pp. 456-488, 2009.

[3] L. J. Moran, R. Pasquali, H. J. Teede, K. M. Hoeger, and R. J. Norman, "Treatment of obesity in polycystic ovary syndrome: a position statement of the Androgen Excess and Polycystic Ovary Syndrome Society," Fertility and Sterility, vol. 92, no. 6, pp. 1966-1982, 2009.

[4] B. O. Yildiz, R. Azziz, and Androgen Excess and PCOS Society, "Ovarian and adipose tissue dysfunction in polycystic ovary syndrome: report of the 4 th special scientific meeting of the Androgen Excess and PCOS Society," Fertility and Sterility, vol. 94, no. 2, pp. 690-693, 2010.

[5] R. Azziz, "Diagnostic criteria for polycystic ovary syndrome: a reappraisal," Fertility and Sterility, vol. 83, no. 5, pp. 1343-1346, 2005.

[6] G. Garruti, R. Depalo, M. G. Vita et al., "Adipose tissue, metabolic syndrome and polycystic ovary syndrome: from pathophysiology to treatment," Reproductive Biomedicine Online, vol. 19, no. 4, pp. 552-563, 2009.

[7] P. Fenichel, C. Rougier, S. Hieronimus, and N. Chevalier, "Which origin for polycystic ovaries syndrome: genetic, environmental or both?," Annales d'endocrinologie, vol. 78, no. 3, pp. 176-185, 2017.

[8] I. R. Ilie and C. E. Georgescu, "Polycystic ovary syndromeepigenetic mechanisms and aberrant microRNA," Advances in Clinical Chemistry, vol. 71, pp. 25-45, 2015.

[9] S. Behboudi-Gandevani, F. Ramezani Tehrani, M. Rostami Dovom et al., "Insulin resistance in obesity and polycystic ovary syndrome: systematic review and meta-analysis of observational studies," Gynecological Endocrinology, vol. 32, no. 5, pp. 343-353, 2016

[10] S. S. Lim, M. J. Davies, R. J. Norman, and L. J. Moran, "Overweight, obesity and central obesity in women with polycystic ovary syndrome: a systematic review and meta-analysis," Human Reproduction Update, vol. 18, no. 6, pp. 618-637, 2012.
[11] P. L. Huang, "A comprehensive definition for metabolic syndrome," Disease Models \& Mechanisms, vol. 2, no. 5-6, pp. 231-237, 2009.

[12] L. Moran and H. Teede, "Metabolic features of the reproductive phenotypes of polycystic ovary syndrome," Human Reproduction Update, vol. 15, no. 4, pp. 477-488, 2009.

[13] L. J. Moran, M. L. Misso, R. A. Wild, and R. J. Norman, "Impaired glucose tolerance, type 2 diabetes and metabolic syndrome in polycystic ovary syndrome: a systematic review and meta-analysis," Human Reproduction Update, vol. 16, no. 4, pp. 347-363, 2010.

[14] L. J. Moran, W. A. March, M. J. Whitrow, L. C. Giles, M. J. Davies, and V. M. Moore, "Sleep disturbances in a community-based sample of women with polycystic ovary syndrome," Human Reproduction, vol. 30, no. 2, pp. 466472, 2015.

[15] B. Chatterjee, J. Suri, J. C. Suri, P. Mittal, and T. Adhikari, "Impact of sleep-disordered breathing on metabolic dysfunctions in patients with polycystic ovary syndrome," Sleep Medicine, vol. 15, no. 12, pp. 1547-1553, 2014.

[16] H. Kahal, I. Kyrou, A. A. Tahrani, and H. S. Randeva, "Obstructive sleep apnoea and polycystic ovary syndrome: a comprehensive review of clinical interactions and underlying pathophysiology," Clinical Endocrinology, vol. 87, no. 4, pp. 313-319, 2017.

[17] K. Nandalike, C. Agarwal, T. Strauss et al., "Sleep and cardiometabolic function in obese adolescent girls with polycystic ovary syndrome," Sleep Medicine, vol. 13, no. 10, pp. 1307-1312, 2012.

[18] M. Barnaś, M. Maskey-Warzęchowska, P. Bielicki, M. Kumor, and R. Chazan, "Diurnal and nocturnal serum melatonin concentrations after treatment with continuous positive airway pressure in patients with obstructive sleep apnea," Polish Archives of Internal Medicine, vol. 127, no. 9, pp. 589-596, 2017.

[19] A. J. R. Lim, Z. Huang, S. E. Chua, M. S. Kramer, and E. L. Yong, "Sleep duration, exercise, shift work and polycystic ovarian syndrome-related outcomes in a healthy population: a cross-sectional study," PLoS One, vol. 11, no. 11, article e0167048, 2016.

[20] E. Spinedi, V. Mariani, M. Bulfon, M. Colombani-Vidal, and H. Scaglia, "Analysis of the hypothalamic-pituitary-ovary axis in the neonatally-androgenized female rat," Journal of Endocrinological Investigation, vol. 13, no. 6, pp. 481-488, 1990.

[21] C. R. McCartney, "Maturation of sleep-wake gonadotrophinreleasing hormone secretion across puberty in girls: potential mechanisms and relevance to the pathogenesis of polycystic ovary syndrome," Journal of Neuroendocrinology, vol. 22, no. 7, pp. 701-709, 2010.

[22] M. Ashwell, S. Chinn, S. Stalley, and J. S. Garrow, "Female fat distribution-a photographic and cellularity study," International Journal of Obesity, vol. 2, no. 3, pp. 289-302, 1978.

[23] W. H. Mueller and S. K. Joos, "Android (centralized) obesity and somatotypes in men: association with mesomorphy," Annals of Human Biology, vol. 12, no. 4, pp. 377-381, 1985.

[24] A. Basdevant, J. Raison, and B. Guy-Grand, "Influence of the distribution of body fat on vascular risk," Presse Médicale, vol. 16, pp. 167-170, 1987.

[25] C. Alexanderson, E. Eriksson, E. Stener-Victorin et al., "Postnatal testosterone exposure results in insulin resistance, 
enlarged mesenteric adipocytes, and an atherogenic lipid profile in adult female rats: comparisons with estradiol and dihydrotestosterone," Endocrinology, vol. 148, no. 11, pp. 5369-5576, 2007.

[26] M. Demissie, M. Lazic, E. M. Foecking, F. Aird, A. Dunaif, and J. E. Levine, "Transient prenatal androgen exposure produces metabolic syndrome in adult female rats," American Journal of Physiology. Endocrinology and Metabolism, vol. 295, no. 2, pp. E262-E268, 2008.

[27] L. Mannerås-Holm, H. Leonhardt, J. Kullberg et al., “Adipose tissue has aberrant morphology and function in PCOS: enlarged adipocytes and low serum adiponectin, but not circulating sex steroids, are strongly associated with insulin resistance," The Journal of Clinical Endocrinology and Metabolism, vol. 96, no. 2, pp. E304-E311, 2011.

[28] C. L. Su, M. Chen, P. N. Zhang, W. Xu, and J. F. Lin, "Effects and mechanism of testosterone on the production of inflammatory cytokines and glucose uptake in coculture of RAW264.7 macrophage and 3T3-L1 adipocytes," Zhonghua Yi Xue Za Zhi, vol. 96, no. 33, pp. 2665-2670, 2016.

[29] R. Singh, J. N. Artaza, W. E. Taylor et al., "Testosterone inhibits adipogenic differentiation in 3T3-L1 cells: nuclear translocation of androgen receptor complex with betacatenin and T-cell factor 4 may bypass canonical Wnt signaling to down-regulate adipogenic transcription factors," Endocrinology, vol. 147, no. 1, pp. 141-154, 2006.

[30] M. McIntosh, D. Hausman, R. Martin, and G. Hausman, "Dehydroepiandrosterone attenuates preadipocyte growth in primary cultures of stromal-vascular cells," American Journal of Physiology-Endocrinology and Metabolism, vol. 275, no. 2, pp. E285-E293, 1998.

[31] R. Singh, J. N. Artaza, W. E. Taylor, N. F. Gonzalez-Cadavid, and S. Bhasin, "Androgens stimulate myogenic differentiation and inhibit adipogenesis in C3H 10T1/2 pluripotent cells through an androgen receptor-mediated pathway," Endocrinology, vol. 144, no. 11, pp. 5081-5088, 2003.

[32] J. Joyner, L. Hutley, and D. Cameron, "Intrinsic regional differences in androgen receptors and dihydrotestosterone metabolism in human preadipocytes," Hormone and Metabolic Research, vol. 34, no. 5, pp. 223-228, 2002.

[33] M. G. Zubiría, J. Vidal-Bravo, E. Spinedi, and A. Giovambattista, "Relationship between impaired adipogenesis of retroperitoneal adipose tissue and hypertrophic obesity: role of endogenous glucocorticoid excess," Journal of Cellular and Molecular Medicine, vol. 18, no. 8, pp. 15491561, 2014.

[34] A. Alzamendi, D. Castrogiovanni, H. H. Ortega, R. C. Gaillard, A. Giovambattista, and E. Spinedi, "Parametrial adipose tissue and metabolic dysfunctions induced by fructose-rich diet in normal and neonatal-androgenized adult female rats," Obesity, vol. 18, no. 3, pp. 441-448, 2010.

[35] L. Ongaro, D. Castrogiovanni, A. Giovambattista, R. C. Gaillard, and E. Spinedi, "Enhanced proinflammatory cytokine response to bacterial lipopolysaccharide in the adult male rat after either neonatal or prepubertal ablation of biological testosterone activity," Neuroimmunomodulation, vol. 18, no. 4, pp. 254-260, 2011.

[36] C. Pantoja, J. T. Huff, and K. R. Yamamoto, "Glucocorticoid signaling defines a novel commitment state during adipogenesis in vitro," Molecular Biology of the Cell, vol. 19, no. 10 , pp. 4032-4041, 2008.

[37] L. Ongaro, A. Giovambattista, and E. Spinedi, "Impact of neonatal manipulation of androgen receptor function on endocrine-metabolic programming in the juvenile female rat," ISRN Endocrinology, vol. 2013, Article ID 181950, 7 pages, 2013.

[38] M. N. Dieudonne, R. Pecquery, A. Boumediene, M. C. Leneveu, and Y. Giudicelli, "Androgen receptors in human preadipocytes and adipocytes: regional specificities and regulation by sex steroids," American Journal of PhysiologyCell Physiology, vol. 274, no. 6, pp. C1645-C1652, 1998.

[39] D. Macut, I. Božić-Antić, J. Bjekić-Macut, and K. Tziomalos, "Management of endocrine disease: polycystic ovary syndrome and nonalcoholic fatty liver disease," European Journal of Endocrinology, vol. 177, no. 3, pp. R145-R158, 2017.

[40] W. S. Dantas, I. H. Murai, L. A. Perandini et al., "Acute exercise elicits differential expression of insulin resistance genes in the skeletal muscle of patients with polycystic ovary syndrome," Clinical Endocrinology, vol. 86, no. 5, pp. 688-697, 2017.

[41] D. Macut, J. Bjekić-Macut, D. Rahelić, and M. Doknić, "Insulin and the polycystic ovary syndrome," Diabetes Research and Clinical Practice, vol. 130, pp. 163-170, 2017.

[42] C. Bañuls, S. Rovira-Llopis, A. Martinez de Marañon et al., "Metabolic syndrome enhances endoplasmic reticulum, oxidative stress and leukocyte-endothelium interactions in PCOS," Metabolism, vol. 71, pp. 153-162, 2017.

[43] C. Moretti, G. Lanzolla, M. Moretti, L. Gnessi, and E. Carmina, "Androgens and hypertension in men and women: a unifying view," Current Hypertension Reports, vol. 19, no. 5, p. 44, 2017.

[44] S. Rodriguez-Cuenca, M. Monjo, A. M. Proenza, and P. Roca, "Depot differences in steroid receptor expression in adipose tissue: possible role of the local steroid milieu," American Journal of Physiology. Endocrinology and Metabolism, vol. 288, no. 1, pp. E200-E207, 2005.

[45] L. Ongaro, N. R. Salvetti, A. Giovambattista, E. Spinedi, and H. H. Ortega, "Neonatal androgenization-induced early endocrine-metabolic and ovary misprogramming in the female rat," Life Sciences, vol. 130, pp. 66-72, 2015.

[46] C. J. Smith and K. K. Ryckman, "Epigenetic and developmental influences on the risk of obesity, diabetes, and metabolic syndrome," Diabetes, Metabolic Syndrome and Obesity: Targets and Therapy, vol. 8, pp. 295-302, 2015.

[47] S. O'Neill and L. O'Driscoll, "Metabolic syndrome: a closer look at the growing epidemic and its associated pathologies," Obesity Reviews, vol. 16, no. 1, pp. 1-12, 2015.

[48] A. Kalsbeek, S. la Fleur, and E. Fliers, "Circadian control of glucose metabolism," Molecular Metabolism, vol. 3, no. 4, pp. 372-383, 2014.

[49] W. Huang, K. M. Ramsey, B. Marcheva, and J. Bass, "Circadian rhythms, sleep, and metabolism," The Journal of Clinical Investigation, vol. 121, no. 6, pp. 2133-2141, 2011.

[50] R. Karthikeyan, G. Marimuthu, D. W. Spence et al., "Should we listen to our clock to prevent type 2 diabetes mellitus?" Diabetes Research and Clinical Practice, vol. 106, no. 2, pp. 182-190, 2014.

[51] E. McFadden, M. E. Jones, M. J. Schoemaker, A. Ashworth, and A. J. Swerdlow, "The relationship between obesity and exposure to light at night: cross-sectional analyses of over 
100,000 women in the Breakthrough Generations Study," American Journal of Epidemiology, vol. 180, no. 3, pp. 245250, 2014.

[52] H. E. Molzof, M. D. Wirth, J. B. Burch et al., "The impact of meal timing on cardiometabolic syndrome indicators in shift workers," Chronobiology International, vol. 34, no. 3, pp. 337-348, 2017.

[53] Y. C. Lin, I. C. Hsieh, and P. C. Chen, "Utilizing the metabolic syndrome component count in workers' health surveillance: an example of day-time vs. day-night rotating shift workers," International Journal of Occupational Medicine and Environmental Health, vol. 28, no. 4, pp. 675-688, 2015.

[54] Y. Kawabe, Y. Nakamura, S. Kikuchi et al., "Relationship between shift work and clustering of the metabolic syndrome diagnostic components," Journal of Atherosclerosis and Thrombosis, vol. 21, no. 7, pp. 703-711, 2014.

[55] T. Arora, M. Z. Chen, A. R. Cooper, R. C. Andrews, and S. Taheri, "The impact of sleep debt on excess adiposity and insulin sensitivity in patients with early type 2 diabetes mellitus," Journal of Clinical Sleep Medicine, vol. 12, no. 5, pp. 673-680, 2016.

[56] D. P. Cardinali, P. Cano, V. Jimenez-Ortega, and A. I. Esquifino, "Melatonin and the metabolic syndrome: physiopathologic and therapeutical implications," Neuroendocrinology, vol. 93, no. 3, pp. 133-142, 2011.

[57] D. P. Cardinali and R. Hardeland, "Inflammaging, metabolic syndrome and melatonin: a call for treatment studies," Neuroendocrinology, vol. 104, no. 4, pp. 382-397, 2017.

[58] D. P. Cardinali and D. E. Vigo, "Melatonin, mitochondria and the metabolic syndrome," Cellular and Molecular Life Sciences, vol. 74, no. 21, pp. 3941-3954, 2017.

[59] H. Tamura, A. Takasaki, T. Taketani et al., "The role of melatonin as an antioxidant in the follicle," Journal of Ovarian Research, vol. 5, no. 1, p. 5, 2012.

[60] H. Tamura, Y. Nakamura, A. Korkmaz et al., "Melatonin and the ovary: physiological and pathophysiological implications," Fertility and Sterility, vol. 92, no. 1, pp. 328-343, 2009.

[61] R. J. Reiter, H. Tamura, D. X. Tan, and X. Y. Xu, "Melatonin and the circadian system: contributions to successful female reproduction," Fertility and Sterility, vol. 102, no. 2, pp. 321-328, 2014.

[62] M. Pan, Y. L. Song, J. M. Xu, and H. Z. Gan, "Melatonin ameliorates nonalcoholic fatty liver induced by high-fat diet in rats," Journal of Pineal Research, vol. 41, no. 1, pp. 79-84, 2006.

[63] I. Stumpf, I. Bazwinsky, and E. Peschke, "Modulation of the cGMP signaling pathway by melatonin in pancreatic beta-cells," Journal of Pineal Research, vol. 46, no. 2, pp. 140-147, 2009.

[64] R. Tarquini, V. Bruni, F. Perfetto, L. Bigozzi, L. Tapparini, and B. Tarquini, "Hypermelatoninemia in women with polycystic ovarian syndrome," The European Journal of Contraception \& Reproductive Health Care, vol. 1, no. 4, pp. 349-350, 1996.

[65] R. Luboshitzky, G. Qupti, A. Ishay, Z. Shen-Orr, B. Futerman, and S. Linn, "Increased 6-sulfatoxymelatonin excretion in women with polycystic ovary syndrome," Fertility and Sterility, vol. 76, no. 3, pp. 506-510, 2001.

[66] R. Luboshitzky, P. Herer, and Z. Shen-Orr, "Urinary 6sulfatoxymelatonin excretion in hyperandrogenic women: the effect of cyproterone acetate-ethinyl estradiol treatment,"
Experimental and Clinical Endocrinology \& Diabetes, vol. 112, no. 2, pp. 102-107, 2004.

[67] D. D. Terzieva, M. M. Orbetzova, M. D. Mitkov, and N. G. Mateva, "Serum melatonin in women with polycystic ovary syndrome," Folia Medica, vol. 55, no. 2, pp. 10-15, 2013.

[68] M. Jain, S. Jain, T. B. Singh, C. Haldar, and P. Jain, "Melatonin and its correlation with testosterone in polycystic ovarian syndrome," Journal of Human Reproductive Sciences, vol. 6, no. 4, pp. 253-258, 2013.

[69] M. L. Dubocovich, P. Delagrange, D. N. Krause, D. Sugden, D. P. Cardinali, and J. Olcese, "International Union of Basic and Clinical Pharmacology. LXXV. Nomenclature, classification, and pharmacology of $G$ protein-coupled melatonin receptors," Pharmacological Reviews, vol. 62, no. 3, pp. 343$380,2010$.

[70] I. Prokopenko, C. Langenberg, J. C. Florez et al., "Variants in MTNR1B influence fasting glucose levels," Nature Genetics, vol. 41, no. 1, pp. 77-81, 2009.

[71] M. A. Matuszek, A. Anton, S. Thillainathan, and N. J. Armstrong, "Increased insulin following an oral glucose load, genetic variation near the melatonin receptor MTNR1B, but no biochemical evidence of endothelial dysfunction in young Asian men and women," PLoS One, vol. 10, no. 7, article e0133611, 2015.

[72] Y. Zhan, C. Li, Q. Gao, J. Chen, S. Yu, and S. G. Liu, "Association between the rs4753426 polymorphism in $M T N R 1 B$ with fasting plasma glucose level and pancreatic $\beta$-cell function in gestational diabetes mellitus," Genetics and Molecular Research, vol. 14, no. 3, pp. 8778-8785, 2015.

[73] M. Tarnowski, D. Malinowski, K. Safranow, V. Dziedziejko, and A. Pawlik, "MTNR1A and MTNR1B gene polymorphisms in women with gestational diabetes," Gynecological Endocrinology, vol. 33, no. 5, pp. 395-398, 2017.

[74] J. Dupuis, DIAGRAM Consortium, C. Langenberg et al., "New genetic loci implicated in fasting glucose homeostasis and their impact on type 2 diabetes risk," Nature Genetics, vol. 42, no. 2, pp. 105-116, 2010.

[75] S. Liao, Y. Liu, Y. Tan et al., "Association of genetic variants of melatonin receptor $1 \mathrm{~B}$ with gestational plasma glucose level and risk of glucose intolerance in pregnant Chinese women," PLoS One, vol. 7, no. 7, article e40113, 2012.

[76] C. Liu, Y. Wu, H. Li et al., "MTNR1B rs10830963 is associated with fasting plasma glucose, $\mathrm{HbA}_{1 \mathrm{C}}$ and impaired beta-cell function in Chinese Hans from Shanghai," BMC Medical Genetics, vol. 11, p. 59, 2010.

[77] X. Song, X. Sun, G. Ma et al., "Family association study between melatonin receptor gene polymorphisms and polycystic ovary syndrome in Han Chinese," European Journal of Obstetrics, Gynecology, and Reproductive Biology, vol. 195, pp. 108-112, 2015.

[78] L. Wang, Y. Wang, X. Zhang et al., "Common genetic variation in MTNR1B is associated with serum testosterone, glucose tolerance, and insulin secretion in polycystic ovary syndrome patients," Fertility and Sterility, vol. 94, no. 6, pp. 2486-2489.e2, 2010.

[79] C. Li, Y. Shi, L. You, L. Wang, and Z. J. Chen, "Association of rs10830963 and rs10830962 SNPs in the melatonin receptor (MTNR1B) gene among Han Chinese women with polycystic ovary syndrome," Molecular Human Reproduction, vol. 17, no. 3, pp. 193-198, 2011. 
[80] C. Li, Y. Shi, L. You, L. Wang, and Z. J. Chen, "Melatonin receptor $1 \mathrm{~A}$ gene polymorphism associated with polycystic ovary syndrome," Gynecologic and Obstetric Investigation, vol. 72, no. 2, pp. 130-134, 2011.

[81] M. K. Kim, E. A. Park, H. J. Kim et al., "Does supplementation of in-vitro culture medium with melatonin improve IVF outcome in PCOS?," Reproductive Biomedicine Online, vol. 26, no. 1, pp. 22-29, 2013.

[82] A. Pacchiarotti, G. Carlomagno, G. Antonini, and A. Pacchiarotti, "Effect of myo-inositol and melatonin versus myo-inositol, in a randomized controlled trial, for improving in vitro fertilization of patients with polycystic ovarian syndrome," Gynecological Endocrinology, vol. 32, no. 1, pp. 69-73, 2016.

[83] V. Tagliaferri, D. Romualdi, and E. Scarinci, "Melatonin treatment may be able to restore menstrual cyclicity in women with PCOS: a pilot study," Reproductive Sciences, vol. 25, no. 2, pp. 269-275, 2017.

[84] S. Stanosz, J. von Mach-Szczypinski, K. Sieja, and J. Kooeciuszkiewicz, "Micronized estradiol and progesterone therapy in primary, preinvasive endometrial cancer (1A/G1) in young women with polycystic ovarian syndrome," The Journal of Clinical Endocrinology and Metabolism, vol. 99, no. 12, pp. E2472-E2476, 2014.

[85] N. Mesri Alamdari, R. Mahdavi, N. Roshanravan, N. Lotfi Yaghin, A. Ostadrahimi, and E. Faramarzi, "A double-blind, placebo-controlled trial related to the effects of melatonin on oxidative stress and inflammatory parameters of obese women," Hormone and Metabolic Research, vol. 47, no. 7, pp. 504-508, 2015.

[86] A. K. Amstrup, T. Sikjaer, S. B. Pedersen, L. Heickendorff, L. Mosekilde, and L. Rejnmark, "Reduced fat mass and increased lean mass in response to 1 year of melatonin treatment in postmenopausal women: a randomized placebocontrolled trial," Clinical Endocrinology, vol. 84, no. 3, pp. 342-347, 2016.

[87] M. Kozirog, A. R. Poliwczak, P. Duchnowicz, M. KoterMichalak, J. Sikora, and M. Broncel, "Melatonin treatment improves blood pressure, lipid profile, and parameters of oxidative stress in patients with metabolic syndrome," Journal of Pineal Research, vol. 50, no. 3, pp. 261-266, 2011.

[88] A. Goyal, P. D. Terry, H. M. Superak et al., "Melatonin supplementation to treat the metabolic syndrome: a randomized controlled trial," Diabetology and Metabolic Syndrome, vol. 6, no. 1, p. 124, 2014.

[89] F. Romo-Nava, D. Alvarez-Icaza González, A. FresánOrellana et al., "Melatonin attenuates antipsychotic metabolic effects: an eight-week randomized, double-blind, parallel-group, placebo-controlled clinical trial," Bipolar Disorders, vol. 16, no. 4, pp. 410-421, 2014.

[90] A. Modabbernia, P. Heidari, R. Soleimani et al., "Melatonin for prevention of metabolic side-effects of olanzapine in patients with first-episode schizophrenia: randomized double-blind placebo-controlled study," Journal of Psychiatric Research, vol. 53, pp. 133-140, 2014.

[91] A. Mostafavi, M. Solhi, M. R. Mohammadi, M. Hamedi, M. Keshavarzi, and S. Akhondzadeh, "Melatonin decreases olanzapine induced metabolic side-effects in adolescents with bipolar disorder: a randomized double-blind placebocontrolled trial," Acta Medica Iranica, vol. 52, no. 10, pp. 734-739, 2014.
[92] V. B. Shatilo, E. V. Bondarenko, and I. A. AntoniukShcheglova, "Pineal gland melatonin-producing function in elderly patients with hypertensive disease: age peculiarities," Advances in Gerontology, vol. 23, no. 4, pp. 539-542, 2010.

[93] M. Gonciarz, Z. Gonciarz, W. Bielanski et al., "The effects of long-term melatonin treatment on plasma liver enzymes levels and plasma concentrations of lipids and melatonin in patients with nonalcoholic steatohepatitis: a pilot study," Journal of Physiology and Pharmacology, vol. 63, no. 1, pp. 35-40, 2012.

[94] M. Gonciarz, Z. Gonciarz, W. Bielanski et al., "The pilot study of 3-month course of melatonin treatment of patients with nonalcoholic steatohepatitis: effect on plasma levels of liver enzymes, lipids and melatonin," Journal of Physiology and Pharmacology, vol. 61, no. 6, pp. 705-710, 2010.

[95] S. A. Hussain, H. M. Khadim, B. H. Khalaf, S. H. Ismail, K. I. Hussein, and A. S. Sahib, "Effects of melatonin and zinc on glycemic control in type 2 diabetic patients poorly controlled with metformin," Saudi Medical Journal, vol. 27, no. 10, pp. 1483-1488, 2006.

[96] C. J. McMullan, G. C. Curhan, E. S. Schernhammer, and J. P. Forman, "Association of nocturnal melatonin secretion with insulin resistance in nondiabetic young women," American Journal of Epidemiology, vol. 178, no. 2, pp. 231-238, 2013.

[97] P. Rubio-Sastre, F. A. J. L. Scheer, P. Gómez-Abellán, J. A. Madrid, and M. Garaulet, "Acute melatonin administration in humans impairs glucose tolerance in both the morning and evening," Sleep, vol. 37, no. 10, pp. 1715-1719, 2014.

[98] R. H. Eckel, C. M. Depner, L. Perreault et al., "Morning circadian misalignment during short sleep duration impacts insulin sensitivity," Current Biology, vol. 25, no. 22, pp. 3004-3010, 2015

[99] K. H. Rubin, D. Glintborg, M. Nybo, B. Abrahamsen, and M. Andersen, "Development and risk factors of type 2 diabetes in a nationwide population of women with polycystic ovary syndrome," The Journal of Clinical Endocrinology and Metabolism, vol. 102, no. 10, pp. 3848-3857, 2017.

[100] H. Ahmadieh and S. Azar, "Effects of sodium glucose cotransporter-2 inhibitors on serum uric acid in type 2 diabetes mellitus," Diabetes Technology \& Therapeutics, vol. 19, no. 9, pp. 507-512, 2017.

[101] K. N. Lew and A. Wick, "Pharmacotherapy of type 2 diabetes mellitus: navigating current and new therapies," Medsurg Nursing, vol. 24, pp. 413-419, 2015.

[102] R. B. Goldberg, J. R. Guyton, T. Mazzone et al., "Relationships between metabolic syndrome and other baseline factors and the efficacy of ezetimibe/simvastatin and atorvastatin in patients with type 2 diabetes and hypercholesterolemia," Diabetes Care, vol. 33, no. 5, pp. 1021-1024, 2010.

[103] M. A. Nauck and N. Friedrich, "Do GLP-1-based therapies increase cancer risk?," Diabetes Care, vol. 36, Supplement 2, pp. S245-S252, 2013.

[104] S. A. Pai and A. S. Majumdar, "Protective effects of melatonin against metabolic and reproductive disturbances in polycystic ovary syndrome in rats," The Journal of Pharmacy and Pharmacology, vol. 66, no. 12, pp. 17101721, 2014.

[105] M. F. Prata Lima, E. C. Baracat, and M. J. Simoes, "Effects of melatonin on the ovarian response to pinealectomy or 
continuous light in female rats: similarity with polycystic ovary syndrome," Brazilian Journal of Medical and Biological Research, vol. 37, no. 7, pp. 987-995, 2004.

[106] F. Nikmard, E. Hosseini, M. Bakhtiyari, M. Ashrafi, F. Amidi, and R. Aflatoonian, "Effects of melatonin on oocyte maturation in PCOS mouse model," Animal Science Journal, vol. 88, no. 4, pp. 586-592, 2017.

[107] A. J. J. M. Lemos, C. A. Peixoto, Á. A. C. Teixeira et al., "Effect of the combination of metformin hydrochloride and melatonin on oxidative stress before and during pregnancy, and biochemical and histopathological analysis of the livers of rats after treatment for polycystic ovary syndrome," Toxicology and Applied Pharmacology, vol. 280, no. 1, pp. 159-168, 2014.

[108] E. S. Pagano, E. Spinedi, and J. J. Gagliardino, "White adipose tissue and circadian rhythm dysfunctions in obesity: pathogenesis and available therapies," Neuroendocrinology, vol. 104, no. 4, pp. 347-363, 2017. 


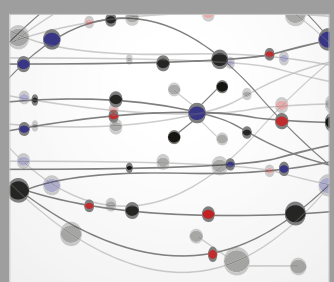

The Scientific World Journal
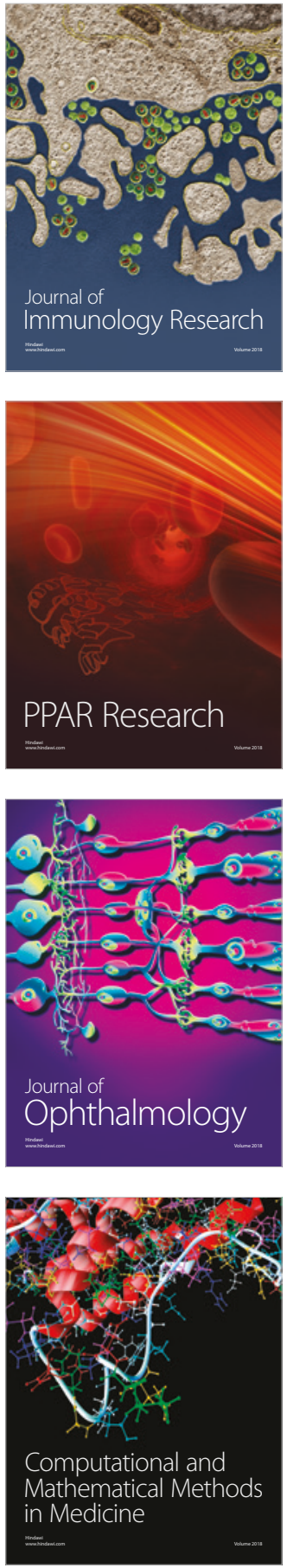

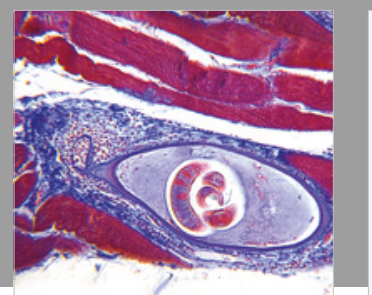

Gastroenterology Research and Practice

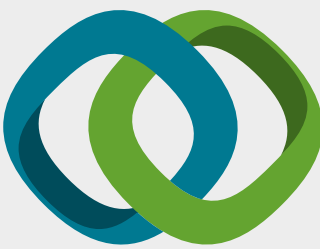

\section{Hindawi}

Submit your manuscripts at

www.hindawi.com
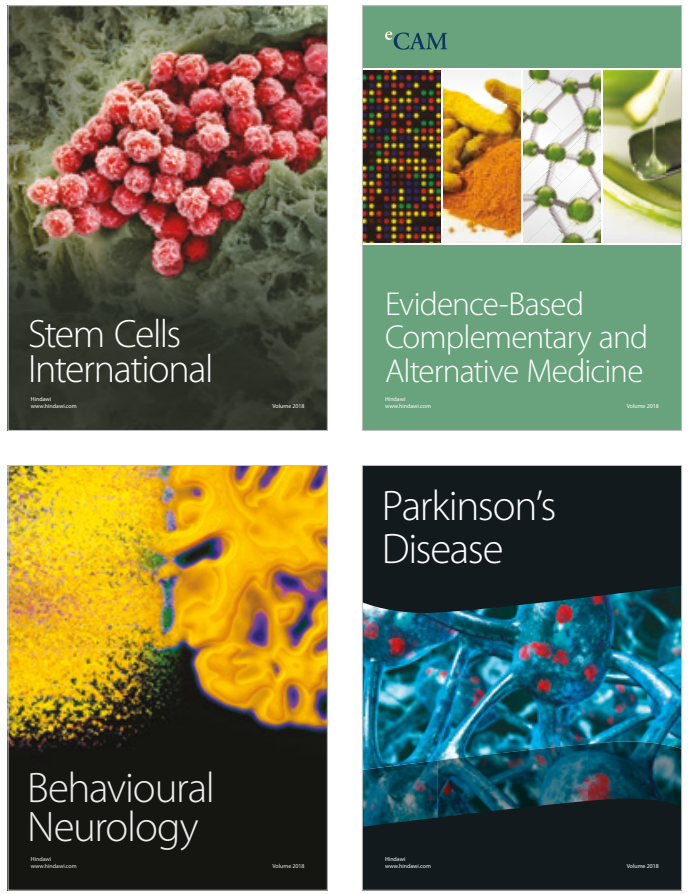

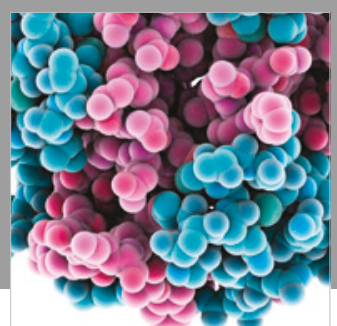

ournal of

Diabetes Research

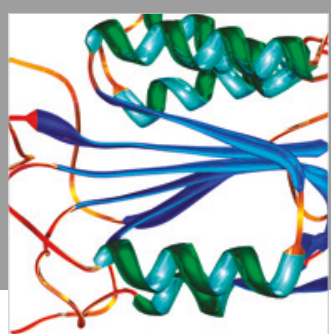

Disease Markers
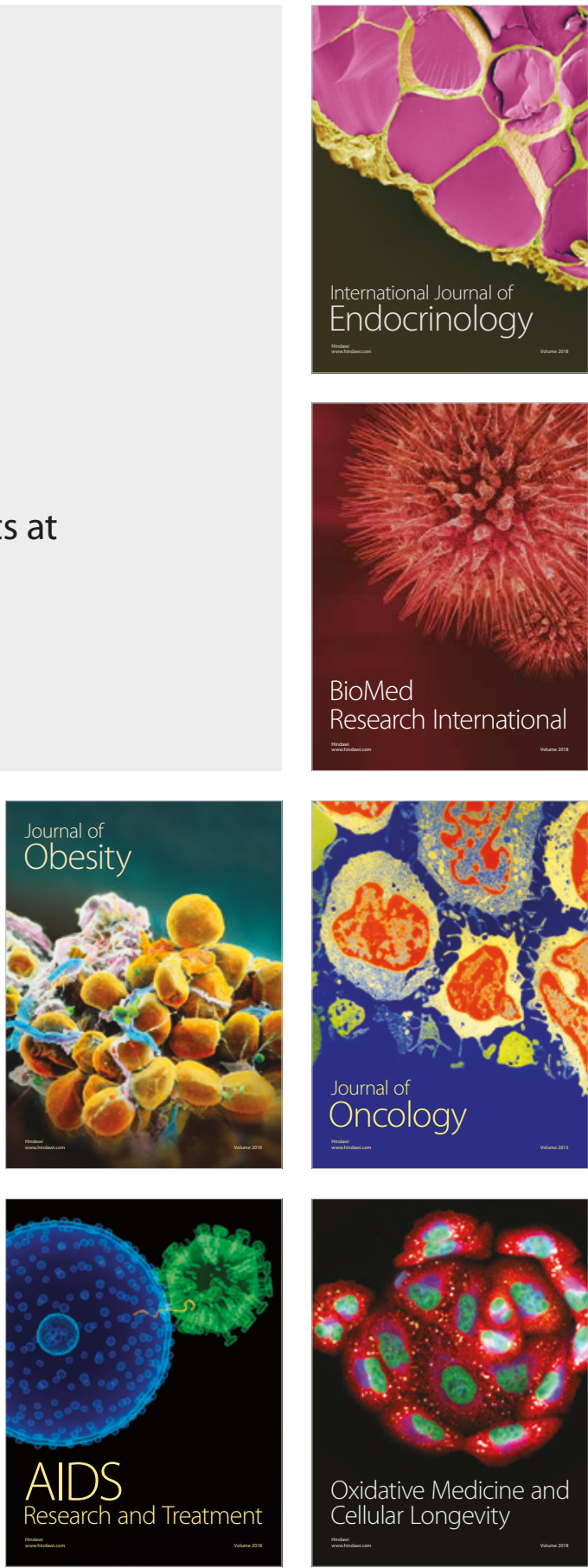